\title{
Effect of an unifaceted and multifaceted intervention on antibiotic prescription control for respiratory diseases: a systematic review of randomized controlled trials
}

Chang Yue ( $\sim 4567401 @ q q . c o m$ )

Guizhou Medical University https://orcid.org/0000-0003-1048-6932

Zhezhe Cui

Guangxi Zhuang Autonomous Region Center for Disease Control and Prevention

Guanghong Yang

Guizhou Medical University

Xun $\mathrm{He}$

Guizhou Medical University

Lei Wang

Guizhou Provincial Health Commission

Zhang Xin

Guizhou Medical University

Lei Tang

Guizhou Medical University

Research

Keywords:

Posted Date: February 12th, 2021

DOl: https://doi.org/10.21203/rs.3.rs-76757/v6

License: (a) (i) This work is licensed under a Creative Commons Attribution 4.0 International License.

Read Full License 


\section{Abstract}

Background The global health system is improperly using antibiotics, particularly in the treatment of respiratory diseases. We aimed to examine the effectiveness of implementing an unifaceted and multifaceted intervention for unreasonable antibiotic prescriptions. Methods Relevant literature published in the databases of Pubmed, Embase, Science Direct, Cochrane Central Register of Controlled Trials (CENTRAL), Chinese Journal Full-text (CNKI) and Wanfang was searched. Data were independently filtered and extracted by two reviewers based on a pre-designed inclusion and exclusion criteria. The Cochrane collaborative bias risk tool was used to evaluate the quality of the included studies. Results $A$ total of 1,074 relevant studies were obtained of which 69 were included in the systematic review. Fiftynine studies reported positive results, that is, the primary results in the intervention groups were superior to those in the control groups. The remaining 10 studies had negative or partially negative results. In 22 studies the outcome variable was the antibiotic prescription rate with detailed reports of the number of prescriptions being further analyzed, of which 19 involved educational interventions for doctors, including: (1) Online training using email, web pages and webinar, (2) Antibiotic guidelines for information dissemination measures by email, postal or telephone reminder, (3) Training doctors in communication skills, (4) Short-term interactive educational seminars, and (5) Short-term field training sessions. Seventeen studies of interventions for health care workers also included: (6) Regular or irregular assessment/audit of antibiotic prescriptions, (7) Prescription recommendations from experts and peers delivered at a meeting or online, (8) Publicly reporting on doctors' antibiotic usage to patients, hospital administrators, and health authorities, (9) Monitoring/feedback prescribing behavior to general practices by email or poster, and (10) Studies involving patients and their families $(n=10)$. Seventeen studies were rated as having a low risk of bias while five were rated as having a high risk of bias. Conclusion The combination of education, prescription audit, prescription recommendations from experts, public reporting, prescription feedback and patient or family member multiple interventions can effectively reduce antibiotic prescription rates in health care institutions. Moreover, adding multiple interventions to educational interventions interventions can control antibiotic prescription rates and may be a more reasonable method. Registrations: This systematic review was registered in PROSPERO, registration number: CRD42020192560. Keywords: Randomized controlled trial, Respiratory diseases, Antibiotic prescriptions, Systematic evaluation 